\title{
Multicolor saccharide-sensing chips created via layer-by- layer adsorption of boronic acid-containing polymers
}

Wakana Takayoshi, Mitsuyuki Imajo, Mizuki Iijima, Marina Suzuki, Hiroki Yamamoto, and Yasumasa Kanekiyo*

Department of Biotechnology and Environmental Chemistry, Kitami Institute of Technology, 165 Koen-cho, Kitami, Hokkaido 090-8507, Japan.

\section{*Corresponding Author}

Tel.: +81-157-26-9389

Fax.: +81-157-26-9389

E-mail: kanekiyo@mail.kitami-it.ac.jp

\begin{abstract}
A novel saccharide-sensing chip that shows a distinct color change was developed. The saccharide-responsive thin films were obtained on a pattern-printed microscope slide via a layerby-layer method utilizing a boronic acid-containing polycation. After adsorption of anionic dyes, the thin films were immersed in aqueous saccharide solutions. As the saccharide concentration increased, the thin films showed a multi-patterned color change that enabled the quantification of saccharides using multi-colorimetric sensing.
\end{abstract}




\section{Keywords}

Sensor, Saccharide, Sugar, Fructose, Boronic acid, Thin film, Colorimetric

\section{Introduction}

Research on saccharide sensors has attracted significant attention because saccharides play a crucial role in the metabolic pathway of living organisms. In recent years, the number of diabetic patients has been increasing remarkably in not only developed countries but also developing countries [1]. To confront this worldwide problem, it is essential to develop a sensitive, durable, inexpensive, and easily operable saccharide sensor for personal diagnosis of diabetes. Since the 1990s, the molecular recognition abilities of boronic acids against saccharides have been extensively studied [2-5]. These studies have proven that boronic acid is a powerful tool for the development of saccharide sensors in aqueous systems. So far, a large number of boronic acidbased saccharide sensors have been developed, such as colorimetric [6-10], fluorescent [11,12], and electrochemical [13-15] sensors. When developing saccharide sensors which can be used by not only specialist but also ordinary people, colorimetric sensors are useful in terms of easy handling. However, sometimes boronic acid-based sensors suffer from a lack of easily observable distinct color change. Thus the introduction of a novel methodology is desirable for the development of practically applicable boronic acid-based sensors.

Our group previously reported the unique behavior of a saccharide-responsive polymer synthesized via the copolymerization of a boronic acid monomer, an amine monomer, acrylamide, and a crosslinker [16]. Subsequently, we have succeeded in developing novel saccharide-sensing chips based on the saccharide-responsive polymer [17]. This sensing chip 
was prepared by polymerizing the above mentioned monomers on a glass plate to form a thin film with saccharide responsiveness. After adsorbing anionic dyes, the sensing chip was immersed in aqueous saccharide solutions. With increasing saccharide concentration, the thin film exhibited a distinct color change resembling a "traffic signal" from green to red via yellow. The mechanism of the color change is believed to involve the formation of negatively charged complexes of the boronic acid units with the saccharides, which results in the replacement of the anionic dyes. Even though the saccharide-responsive color change is quite unique, it suffers from a relatively long response time $(\sim 1 \mathrm{~h})$. To realize practical saccharide sensors, it is desirable to shorten the response time.

Herein we report our recent results regarding the development of a rapidly responsive saccharide-sensing chip that exhibits multicolor changes. To achieve this, the film thickness was reduced by applying the layer-by-layer adsorption technique [18-21] for the preparation of the saccharide-responsive thin film. After the preparation of the layer-by-layer thin films on a pattern-printed microscope slide, each film was stained using anionic dyes of different colors. The color change of the thin films was then examined upon exposure to saccharides.

\section{Experimental}

\subsection{Materials}

Anionic dyes (1-6) were purchased from Tokyo Chemical Industries (Tokyo, Japan). Sodium polyacrylate (degree of polymerization: 22,000-70,000) was purchased from Wako Pure Chemicals Industries (Osaka, Japan). 3-Acrylamidophenylboronic acid (7) was synthesized according to the literature method [22]. N-(3-aminopropyl) methacrylamide hydrochloride (8) was purchased from Funakoshi (Tokyo, Japan). Other reagents were purchased from Wako. All 
chemicals were reagent grade and used as received. Pattern-printed microscope slide was purchased from Matsunami Glass Industries (Osaka, Japan).

\subsection{Synthesis of boronic acid-containing polycation}

Copolymerization was carried out in a homogeneous solution containing boronic acid monomer 7, amine monomer 8 , and ammonium persulfate $(12 \mathrm{mg}, 54 \mu \mathrm{mol})$ in a mixed solvent of water and methanol $(2 \mathrm{~mL}+2 \mathrm{~mL})$ at $60{ }^{\circ} \mathrm{C}$ for 1 night. Compositions of the monomer mixtures are shown in Table 1. After the polymerization, the reaction mixture was poured in a large amount of 2-propanol and the obtained precipitate was thoroughly washed with acetone. After that, the copolymer was dried under vacuum. Compositions of the obtained polycations were determined by measuring ${ }^{1} \mathrm{H}$ NMR spectra in $\mathrm{D}_{2} \mathrm{O}$ (Figure $\mathrm{S} 8$ ) as follows: boronic acid unit / amine unit = 1:0.99 (polycation A), 1:1.80 (polycation B), 1:2.64 (polycation C).

\subsection{Preparation of layer-by-layer thin films}

Boronic acid-containing polycation and sodium polyacrylate were respectively dissolved in aqueous solutions buffered at $\mathrm{pH} 9.5$ (adjusted by $100 \mathrm{mM}$ ammonium buffer). Concentrations of the polymers were as follows: [polycation] $=1.37 \mathrm{mg} \mathrm{mL}^{-1}$, [polyanion] $=1.03 \mathrm{mg} \mathrm{mL}^{-1}$. Firstly, $0.1 \mathrm{~mL}$ of polycation solution was poured on the glass surface of a pattern-printed microscope slide. After 10 min, the glass surface was washed with water and then dried by air. Then, $0.1 \mathrm{~mL}$ of polyanion solution was poured on the surface and allowed to stand for $10 \mathrm{~min}$. After that, the glass surface was washed with water and then dried by air. These procedures were repeated 10 times, and finally polycation was adsorbed on the surface. It is defined that one 
adsorption cycle (polycation + polyanion) forms 1 layer, so that the resultant film consisted of 10.5 layers. The layer-by-layer process was conducted at room temperature.

\subsection{Staining of thin films}

Anionic dye solutions $(0.1 \mathrm{~mL}$ each) were respectively poured onto each spot on the patternprinted microscope slide on which the layer-by-layer thin films had been prepared at $25{ }^{\circ} \mathrm{C}$. Concentrations of the dye solutions were as follows: $1 \mathrm{mM}$ Acid Blue 92 (Spot 1), $1 \mathrm{mM}$ Brilliant Yellow (Spot 2), $1 \mathrm{mM}$ Sunset Yellow FCF (Spot 3), $0.2 \mathrm{mM}$ Indigotrisulfonate (Spot 4), $0.18 \mathrm{mM}$ Tartrazine (Spot 5), $1 \mathrm{mM}$ Acid Red 27 (Spot 6). After $1 \mathrm{~h}$, the thin films were washed with water and dried by air. Then, the same procedure for dye adsorption was repeated twice. For the adjustment of the solution $\mathrm{pH}, 10 \mathrm{mM}$ phosphate buffer $(\mathrm{pH} 7.0-7.8)$ and $10 \mathrm{mM}$ ammonium buffer ( $\mathrm{pH}$ 9.5) were used.

\subsection{Response against saccharide}

The sensing chips were immersed in $50 \mathrm{~mL}$ of aqueous fructose solutions at $\mathrm{pH} 7.4$ (adjusted by $10 \mathrm{mM}$ phosphate buffer) and $25{ }^{\circ} \mathrm{C}$. The time-resolved color change in each spot was observed by measuring UV-Vis absorption spectra of the film at normal incidence using an integrating sphere.

\subsection{Apparatus}

UV-Vis absorption spectra were obtained by using JASCO V-650 equipped with integrating sphere ISV-722. Scanning electron microscope (SEM) images were obtained by JEOL JSM6701F. Quartz crystal microbalance (QCM) measurements were conducted with Initiam 
AFFINIX QN. Determination of $\mathrm{pH}$ values were made with Metrohm 827pH lab. NMR spectra were measured with JEOR JNM-ECA600. Aqueous solutions were prepared with distilled water purified by Yamato WG202 system.

\section{Results and discussion}

\subsection{Preparation of saccharide sensing chip}

A boronic acid-containing polycation (Polycation B, Fig. 1) and a polyanion (sodium polyacrylate) were alternately adsorbed on the glass surface of a pattern-printed microscope slide at $\mathrm{pH} 9.5$ using a layer-by-layer technique. The procedure is illustrated in Fig. 2. The microscope slide was covered with a hydrophobic coating ( $20 \mu \mathrm{m}$ in thickness) having circular holes $(8 \mathrm{~mm}$ in diameter) that left the glass surface exposed. First, the aqueous polycation solution was poured onto the glass surface. After $10 \mathrm{~min}$, the glass surface was washed with water and dried in air. Next, the polyanion solution was poured onto the surface for $10 \mathrm{~min}$ and then washed with water and dried in air. This adsorption cycle was repeated until 10.5 layers $(1$ cycle $=1$ layer $)$ were adsorbed. The film thickness was determined to be $c a$. $1000 \mathrm{~nm}$ via scanning electron microscopy (SEM) analysis of a cross-section of the thin film (Fig. 3). Since the film thickness prepared by a polymerization method was $c a .10 \mu \mathrm{m}$ [17], it can be said that we were able to reduce film thickness to $1 / 10$ by using the layer-by-layer method. The layer-by-layer growth of the film was monitored by taking measurements with a quartz crystal microbalance (QCM), as shown in Fig. 4. When the layer-by-layer film was grown on a QCM resonator surface, the thickness per layer was relatively small during the early stage of growth, but then gradually increased with the layer number. After the adsorption of 10.5 layers, the film thickness was calculated to be $978 \mathrm{~nm}$ based on the resonant frequency change, which agreed with the SEM 
measurement. It is important to note that the sum of the thickness of the polycation layers was evaluated to be $900 \mathrm{~nm}$, while that of the polyanion layers was much smaller $(78 \mathrm{~nm})$.

The resultant thin films were stained using various anionic dyes (Fig. 5). First, the effect of $\mathrm{pH}$ on the adsorption of the anionic dyes onto the thin films was evaluated measuring UV spectrum of each film. Absorbance derived from the adsorbed anionic dyes markedly changed depending on $\mathrm{pH}$ (see Fig. 6). When the $\mathrm{pH}$ of the anionic dye solutions was 9.5, the thin films were not stained at all. In contrast, the anionic dyes significantly adsorbed onto the thin films at neutral $\mathrm{pH}$ range, and the amount of adsorbed dye tended to increase as the $\mathrm{pH}$ decreased. It is known that boronic acids dissociate to be negatively-charged boronate forms with the $\mathrm{p} K_{\mathrm{a}}$ value of $c a .9$. Therefore, most of the boronic acid units are supposed to exist as an ionized form during the layer-by-layer process conducted at $\mathrm{pH}$ 9.5. Inside the layer-by-layer thin film, charges on the polyelectrolytes were neutralized each other and no electrostatic interaction with the anionic dyes was expected at $\mathrm{pH}$ 9.5. However, when the $\mathrm{pH}$ was lowered to neutral range, the thin film should become positively-charged due to neutralization of the boronate groups. As a result, the anionic dyes were adsorbed onto the thin film through electrostatic interactions.

We examined the effect of monomer composition by using three kinds of polycations having different feed ratios. For the feed ratio of boronic acid monomer $\mathbf{7}$ and amine monomer $\mathbf{8}$, refer to Table 1. Polycation A was not soluble in an aqueous solution at $\mathrm{pH} 9.5$ probably due to low cationic charge density in the polymer. Polycation $\mathrm{C}$ was utilized for the layer-by-layer process and the resultant thin films were stained with anionic dyes. However, absorbances derived from the adsorbed dyes were very small (Indigotrisulfonate: 0.268 , Tartrazine: 0.223 ). This result suggests that the cationic charge density in the layer-by-layer film was much lower than the case 
of Polycation B. Accordingly, we decided to use the thin film prepared with Polycation B for the subsequent experiments.

\subsection{Response against fructose}

The thin films stained at $\mathrm{pH} 7.4$ were subsequently immersed in aqueous $\mathrm{pH} 7.4$ fructose solutions with concentrations ranging from 0 to $100 \mathrm{mM}$. The color of each spot changed with time due to the release of the anionic dyes from the thin films. The time course of the color change is shown in Fig. 7. After $10 \mathrm{~min}$, the color of the sensing chip distinctly changed depending on the fructose concentration. It is known that the apparent $\mathrm{p} K_{\mathrm{a}}$ of boronic acid becomes lower by the interaction with polyhydroxy compounds, because the anionic complexes are much more stable than the corresponding neutral complexes. Therefore, we believe that the boronic acid unit in the thin film gained negative charge and thus the anionic dyes were expelled out of the film [5]. It can be clearly seen that the color change for each spot occurred at different fructose concentration range. When the fructose concentration was $1 \mathrm{mM}$, only Spot 5 showed a sharp color change from yellow to colorless. From 3 to $10 \mathrm{mM}$, Spot 3 gradually became colorless and Spot 4 exhibited a visible discoloration. At $30 \mathrm{mM}$, Spot 1 and 2 were still colored and the other spots were colorless. All the spots finally became colorless at $100 \mathrm{mM}$.

To quantitatively evaluate the color change, absorption spectra of the thin films were measured (see Supplementary data, Fig. S1-S6), and the relative absorbance changes were plotted against the fructose concentration as shown in Fig. 8. Tartrazine (Spot 5) showed the highest responsiveness and became nearly completely released from the thin film when the fructose concentration was $3 \mathrm{mM}$. In contrast, Brilliant Yellow (Spot 2) remained bound until the fructose concentration increased significantly and about half of the dye still remained bound at 
$30 \mathrm{mM}$. The other dyes showed intermediate responsiveness. When the fructose concentration was $10 \mathrm{mM}$, a large part of Sunset Yellow FCF (Spot 3) was removed. At $30 \mathrm{mM}$, Indigotrisulfonate (Spot 4) and Acid Red 27 (Spot 6) were nearly removed, however, some part of Acid Blue 92 (Spot 1) still remained bound. All the dyes were removed at $100 \mathrm{mM}$. This behavior is likely due to the difference in the binding affinity of the anionic dyes to the thin film. In other words, loosely bound dyes are desorbed at lower fructose concentrations, whereas tightly bound dyes are desorbed at higher fructose concentrations. Because brilliant yellow is a divalent anionic dye, the electrostatic interaction with the thin film would be expected to be weaker than that of the trivalent anionic dyes. However, the observed release profile indicates that brilliant yellow is more tightly bound by the thin film than the case of trivalent dyes such as tartrazine. Thus, we speculate that not only electrostatic interactions but also hydrophobic interactions play an important role in determining the binding strength of the dyes. Tartrazine has only two benzene rings, whereas brilliant yellow possesses four benzene rings. Therefore, brilliant yellow must be more hydrophobic than tartrazine, leading to a higher binding affinity with the thin film.

\subsection{Response selectivity}

To compare the response selectivity for different saccharides, the thin films were immersed in $10 \mathrm{mM}$ glucose and sucrose solutions for $10 \mathrm{~min}$. Relative absorbance change for each spot is shown in Fig. 9, and photographs of the resultant sensing chips are shown in Fig. S7. Compared to the results for fructose, the response to glucose was significantly reduced, and sucrose induced virtually no visible color change. It is known that the binding affinity of saccharides to boronic acid is in the following order: fructose $>$ glucose $>$ sucrose [23]. Because this order coincides 
with the observed response selectivity, it can be concluded that the interaction between the boronic acid units in the thin films and the saccharides is the principal driving force for the response.

\section{Conclusions}

In this paper, we established a method for obtaining saccharide-sensing chips that exhibit rapid and distinct color changes. By a layer-by-layer method with a boronic acid-containing polycation, saccharide-responsive thin films were generated on a pattern-printed microscope slide. After the adsorption of anionic dyes, the thin films were immersed in aqueous fructose solutions. As the fructose concentration increased, each spot clearly changed its color. Because the color change occurred at different fructose concentration ranges depending on the anionic dye, the sensing chip exhibited various patterns for the color change. This pattern formation would be useful for the measurement of saccharide concentrations through pattern-based sensing. The plausible mechanism for this sensor is shown in Fig. 10.

\section{Acknowledgements}

This research was supported by a Grant-in-Aid for Scientific Research [KAKENHI (23550088)] from the Ministry of Education, Culture, Sports, Science and Technology of Japan (MEXT). This research was also supported by the Adaptable and Seamless Technology transfer Program through target-driven R\&D (A-STEP) from the Japan Science and Technology Agency (JST).

\section{Appendix A. Supplementary data}




\section{References}

[1] International Diabetes Federation, 2012. IDF Diabetes Atlas 5th ed. http://www.idf.org/diabetesatlas.

[2] T. D. James, K. R. A. S. Sandanayake, S. Shinkai, Angew. Chem., Int. Ed. 35 (1966) 19111922.

[3] M. Bielecki, H. Eggert, J. C. Norrild, J. Chem. Soc., Perkin Trans. 2 (1999) 449-455.

[4] G. Springsteen, B. Wang, Tetrahedron 58 (2002) 5291-5300.

[5] T. D. James, M. D. Phillips, S. Shinkai, Boronic Acids in Saccharide Recognition, RSC Publishing, Cambridge, UK, 2006.

[6] N. DiCesare, J. R. Lakowicz, Org. Lett. 3 (2001) 3891-3893.

[7] S. Kabilan, A. J. Marshall, F. K. Sartain, M.-C. Lee, A. Hussain, X. Yang, J. Blyth, N. Karangu, K. James, J. Zeng, D. Smith, A. Domschke, C. R. Lowe, Biosens. Bioelectron. 20 (2005) 1602-1610.

[8] Y. Anraku, Y. Takahashi, H. Kitano, M. Hakari, Colloids Surf., B 57 (2007) 61-68.

[9] C. Shimpuku, R. Ozawa, A. Sasaki, F. Sato, T. Hashimoto, A. Yamauchi, I. Suzuki, T. Hayashita, Chem. Commun. 45 (2009) 1709-1711.

[10]M. Tsuchiya, Y. Kanekiyo, Analyst 136 (2001) 2521-2526.

[11]H. Fang, G. Kaur, B. Wang, J. Fluoresc. 14 (2004) 481-489.

[12]Y. Kanekiyo, H. Sato, H. Tao, Macromol. Rapid Commun. 26 (2005) 1542-1546. 
[13]S. Takahashi, J. Anzai, Langmuir 21 (2005) 5102-5107.

[14]A. Tiwari, D. Terada, C. Yoshikawa, H. Kobayashi, Talanta 82 (2010) 1725-1732.

[15] J. Li, Z. Wang, P. Li, N. Zong, F. Li, Sensor Actuat. B-Chem. 161 (2012) 832-837.

[16]Y. Kanekiyo, T. Yokozawa, H. Tao, Chem. Lett. 37 (2008) 626-627.

[17] Y. Iwami, T. Yokozawa, W. Takayoshi, Y. Kanekiyo, Talanta 85 (2011) 829-833.

[18] G. Decher, J. Schmit, Thin Solid Films 210/211 (1992) 831-835.

[19] I. Ichinose, H. Tagawa, S. Suzuki, Y. Lovov, T. Kunitake, Langmuir 14 (1998) 187-192.

[20] S. Shiratori, M. F. Rubner, Macromolecules 33 (2000) 4213-4219.

[21]K. Ariga, J. P. Hill, Q. Ji, Phys. Chem. Chem. Phys. 9 (2007) 2319-2340.

[22]Y. Kanekiyo, M. Sano, R. Iguchi, S. Shinkai, J. Polym. Sci., Part A: Polym. Chem. 38 (2000) 1302-1310.

[23] J. P. Lorand, J. O. Edwards, J. Org. Chem. 24 (1959) 769-774. 


\section{Biographies}

Wakana Takayoshi was born in 1987. She received her B. Eng. and M. Eng. from Kitami Institute of Technology, Japan, in 2010 and 2012, respectively.

Mitsuyuki Imajo was born in 1989. He received his B. Eng. from Kitami Institute of Technology in 2012.

Mizuki Iijima was born in 1990. He has been an under graduate student at Kitami Institute of Technology since 2009 .

Marina Suzuki was born in 1989. She received her B. Eng. from Kitami Institute of Technology in 2012. She is currently a graduate student in Kitami Institute of Technology.

Hiroki Yamamoto was born in 1990. He received his B. Eng. from Kitami Institute of Technology in 2013. He is currently a graduate student in Kitami Institute of Technology.

Yasumasa Kanekiyo was born in 1970. He received his B. Sci. and M. Sci. from Nagoya University, Japan, in 1993 and 1995, respectively. He obtained his $\mathrm{PhD}$ in chemistry from Kyushu University, Japan, in 2001. Since 2006, he has been an associate professor at Kitami Institute Technology, Japan. His research interests are molecular recognition chemistry, analytical chemistry, and chemical sensors. 
Fig. 1. Synthesis of boronic acid-containing polycation.

Fig. 2. Preparation of saccharide-sensing chip.

Fig. 3. Cross-sectional SEM image of the thin film.

Fig. 4. Increase in the film thickness during the layer-by-layer process measured by QCM.

Fig. 5. Molecular structures of the investigated anionic dyes. 1: Acid Blue 92, 2: Brilliant Yellow, 3: Sunset Yellow FCF, 4: Indigotrisulfonate, 5: Tartrazine, 6: Acid Red 27.

Fig. 6. Effect of $\mathrm{pH}$ on the adsorption of anionic dyes onto the thin films. Absorbances were observed at $425 \mathrm{~nm}$ (Tartrazine) and $610 \mathrm{~nm}$ (Indigotrisulfonate).

Fig. 7. Color changes on the sensing chip after immersion in aqueous fructose solutions buffered at $\mathrm{pH} 7.4(10 \mathrm{mM}$ phosphate $)$ at $25^{\circ} \mathrm{C}$.

Fig. 8. Absorbance change for each spot as a function of the fructose concentration after immersion in aqueous fructose solutions buffered at $\mathrm{pH} 7.4(10 \mathrm{mM}$ phosphate $)$ at $25{ }^{\circ} \mathrm{C}$ for 10 min. Spot 1: Acid Blue 92 (585 nm), 2: Brilliant Yellow (416 nm), 3: Sunset Yellow FCF (482 nm), 4: Indigotrisulfonate $(610 \mathrm{~nm})$, 5: Tartrazine $(425 \mathrm{~nm})$, 6: Acid Red $27(530 \mathrm{~nm})$.

Fig. 9. Comparison of the responsiveness to various $10 \mathrm{mM}$ saccharide solutions buffered at $\mathrm{pH}$ $7.4\left(10 \mathrm{mM}\right.$ phosphate) at $25^{\circ} \mathrm{C}$ for $10 \mathrm{~min}$.

Fig. 10. Plausible mechanism for this sensor: 1) formation of layer-by-layer film, 2) adsorption of anionic dye, 3) release of dye in response to saccharide.

Table 1. Monomer compositions for the synthesis of polycations. 
Table 1

\begin{tabular}{lll}
\hline & $\mathbf{7}$ & $\mathbf{8}$ \\
\hline Polycation A & $230 \mathrm{mg}(1.2 \mathrm{mmol})$ & $214 \mathrm{mg}(1.2 \mathrm{mmol})$ \\
\hline Polycation B & $115 \mathrm{mg}(0.6 \mathrm{mmol})$ & $214 \mathrm{mg}(1.2 \mathrm{mmol})$ \\
\hline Polycation C & $77 \mathrm{mg}(0.4 \mathrm{mmol})$ & $214 \mathrm{mg}(1.2 \mathrm{mmol})$ \\
\hline
\end{tabular}


Fig. 1

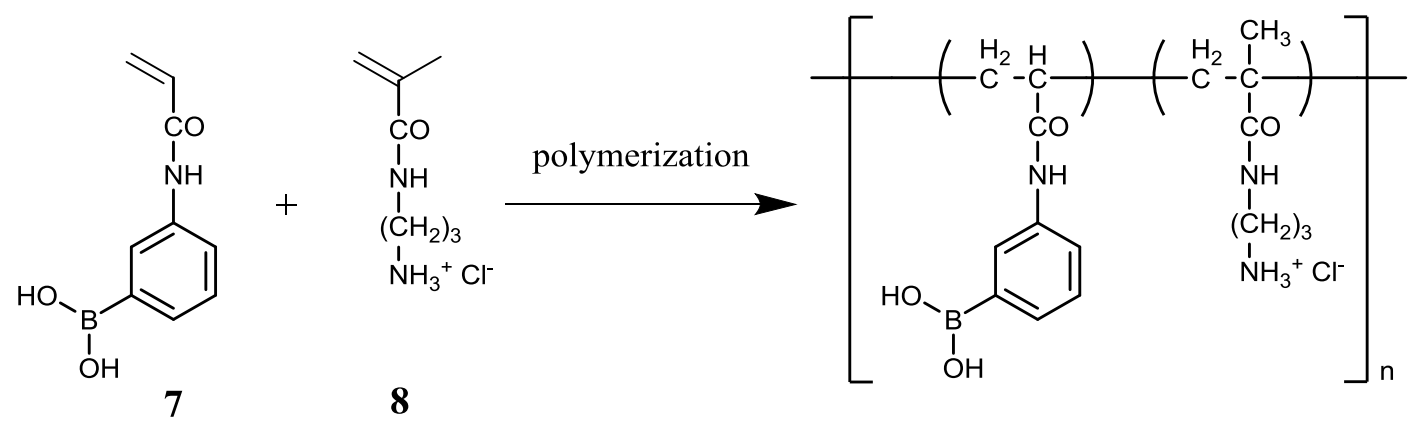


Fig. 2

for Web

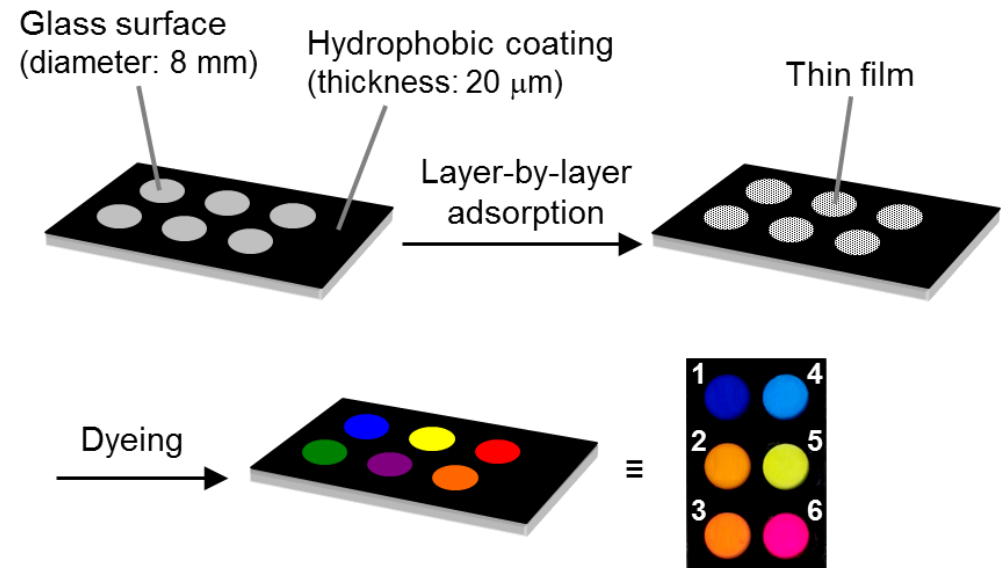

$\underline{\text { for print }}$

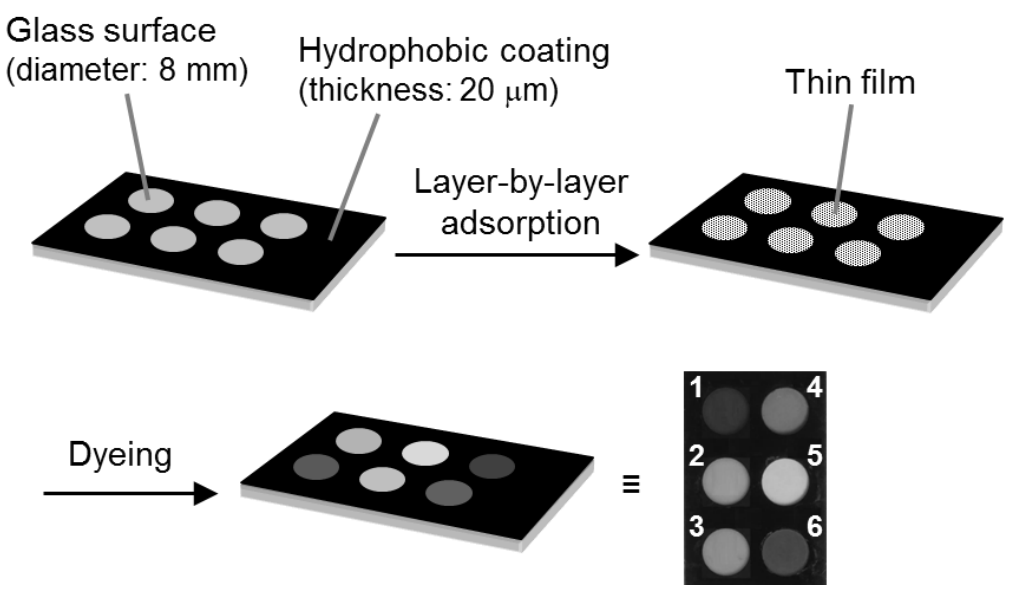


Fig. 3

Film Glass

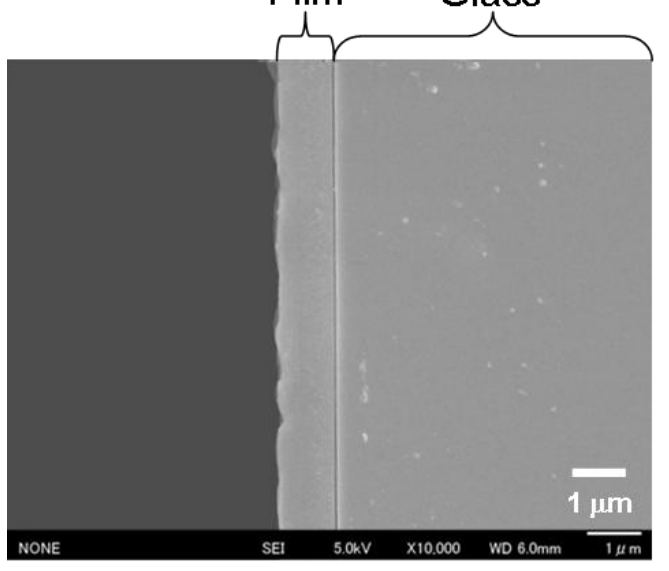


Fig. 4

for Web

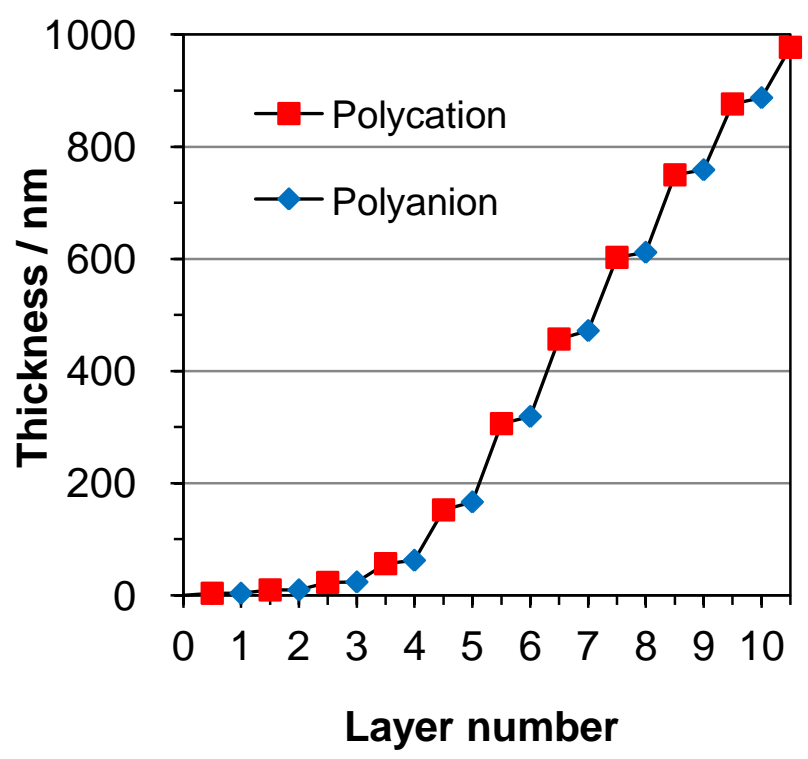

for print

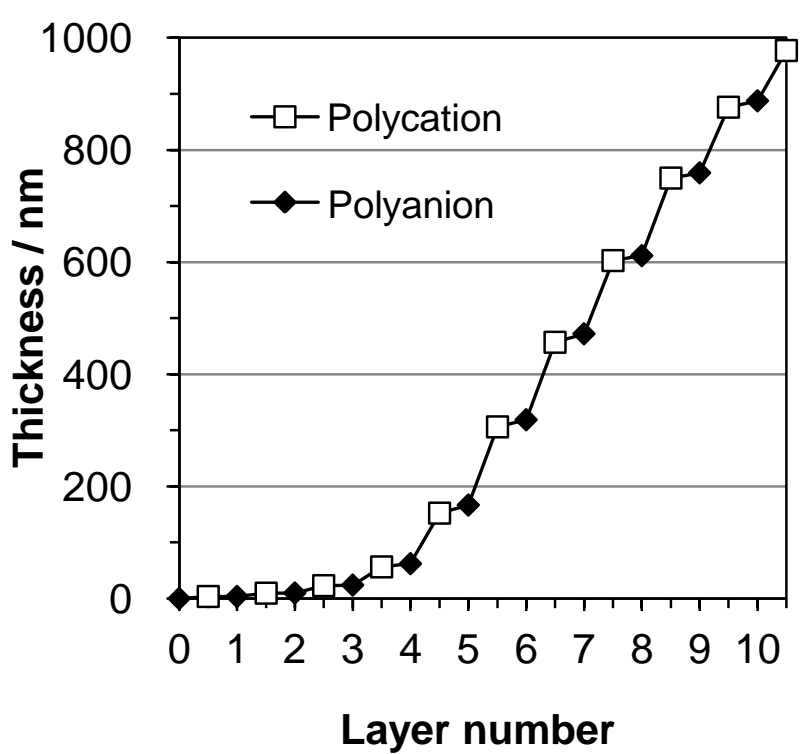


Fig. 5<smiles>O=[S+]([O-])c1cccc2c(N=Nc3cc(S(=O)(=O)[O-])cc4cc([S+](=O)(=O)[O-])cc(O)c34)ccc(Nc3ccccc3)c12</smiles><smiles>O=C1/C(=C2\Nc3c(cc([Se](=O)(=O)[O-])cc3S(=O)(=O)[O-])C2=O)Nc2ccc(S(=O)(=O)[O-])cc21</smiles><smiles>N=Nc1ccc(O)cc1</smiles><smiles>O=S(=O)(O)c1ccc(/N=N/c2c(O)ccc3cc([S+](=O)([O-])[O-])ccc23)cc1</smiles><smiles>O=[N+]([O-])c1ccc(N=Nc2c(O)c([Se](=O)(=O)[O-])cc3cc(S(=O)(=O)O)ccc23)c2ccccc12</smiles> 
Fig. 6

for Web

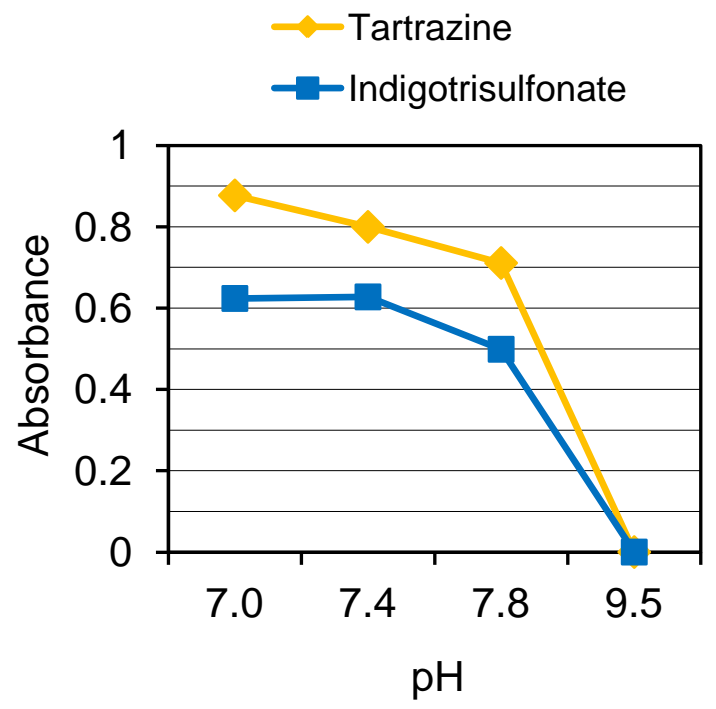

for print

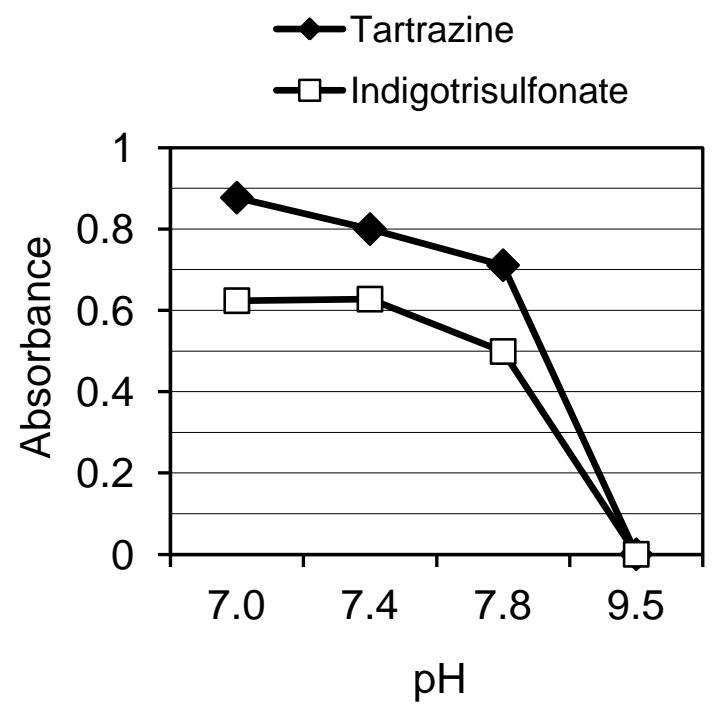


Fig. 7

for Web
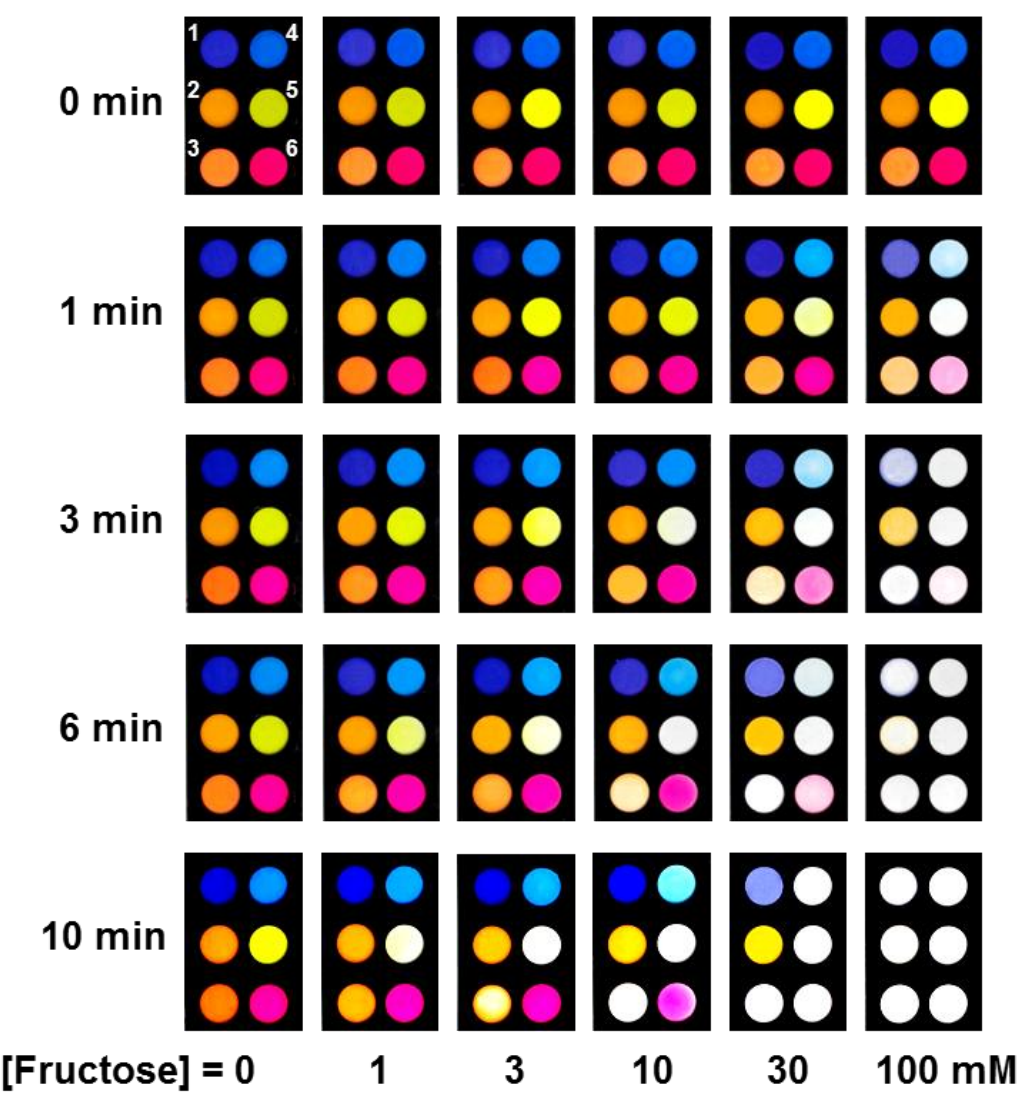
Fig. 7

for print
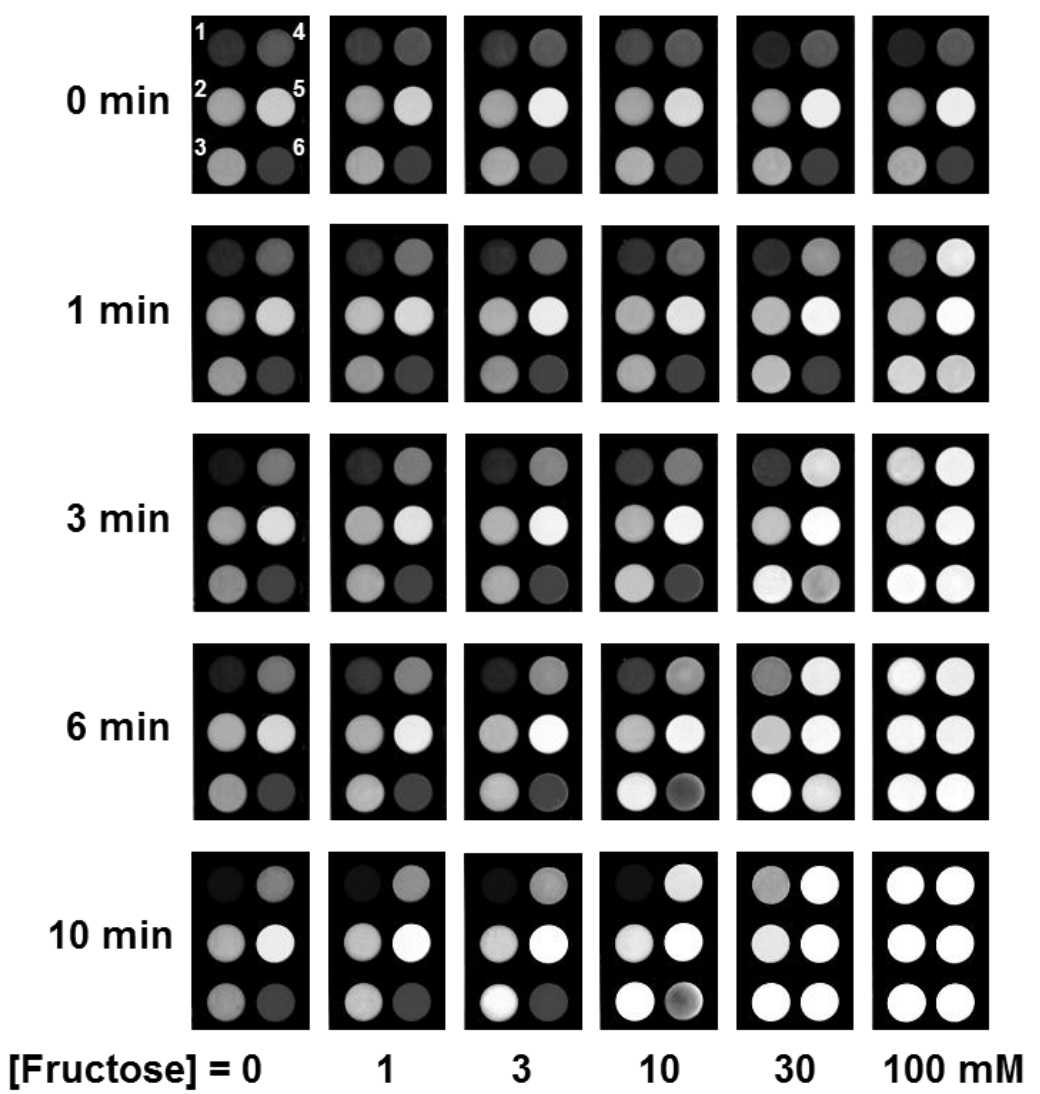
Fig. 8

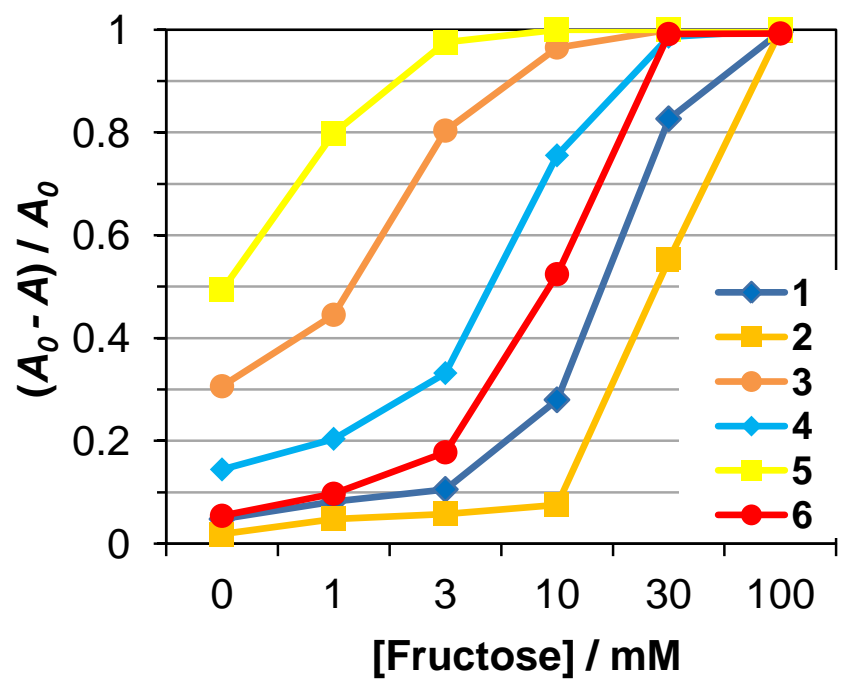


Fig. 9

- Sucrose $\square$ Glucose $\square$ Fructose

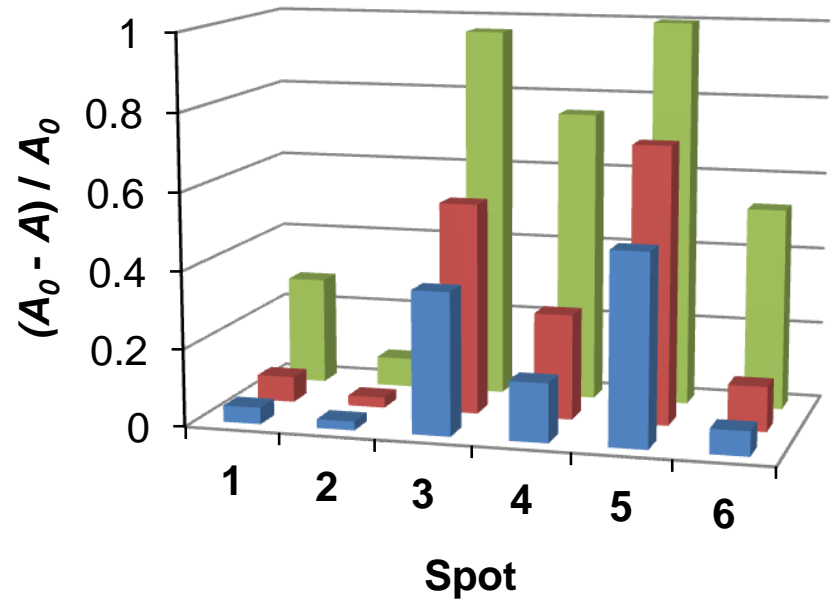


Fig. 10

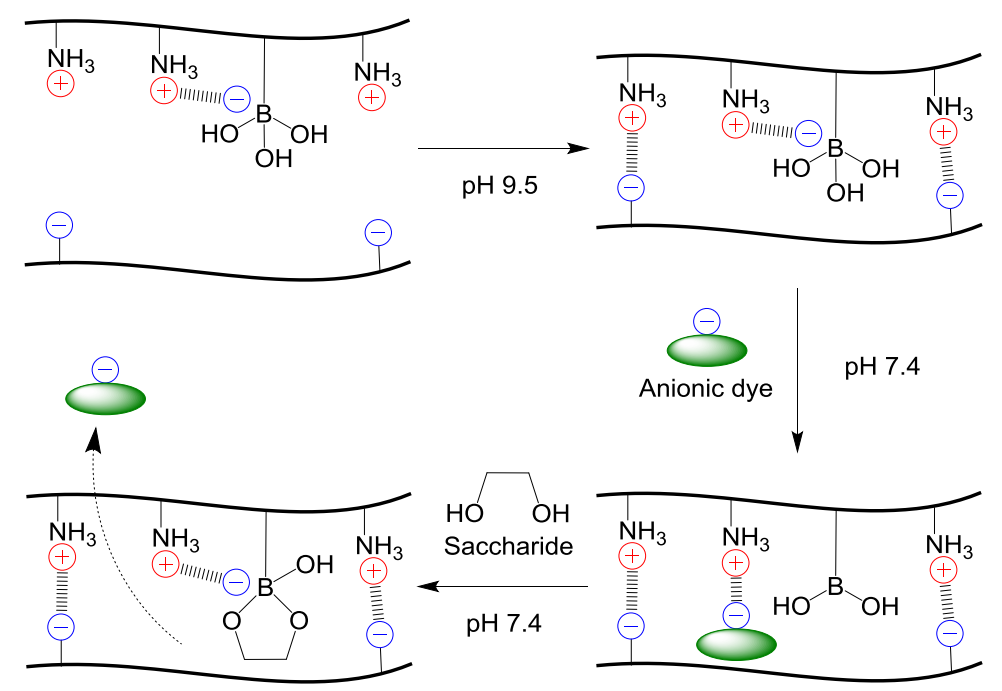

